\title{
Stochastic solutions for time-fractional heat equations with complex spatial variables
}

\author{
Luisa Beghin $^{1}$ - Alessandro De Gregorio ${ }^{1}$
}

Received: 19 September 2021 / Revised: 17 November 2021 / Accepted: 30 November 2021 /

Published online: 22 February 2022

(c) The Author(s) 2022

\begin{abstract}
We deal with complex spatial diffusion equations with time-fractional derivative and study their stochastic solutions. In particular, we complexify the integral operator solution to the heat-type equation where the time derivative is replaced with the convolution-type generalization of the regularized Caputo derivative. We prove that this operator is solution of a complex time-fractional heat equation with complex spatial variable. This approach leads to a wrapped Brownian motion on a circle time-changed by the inverse of the related subordinator. This time-changed Brownian motion is analyzed and, in particular, some results on its moments, as well as its construction as weak limit of continuous-time random walks, are obtained. The extension of our approach to the higher dimensional case is also provided.
\end{abstract}

Keywords Complex singular integrals · Complex evolution equations · Generalized Caputo derivative . Time-changed processes - Wrapped Brownian motion

Mathematics Subject Classification 26A33 - 60G22

\section{Introduction}

The study of the evolution equations with complex spatial variables is a quite recent research topic in the theory of the partial differential equations and complex analysis. In the pioneering works $[8,9]$, the authors proposed two different methods to complexify the spatial variable appearing in different evolution equations (and keeping the time variable real). In particular, for the heat equation a possible approach consists in the

Luisa Beghin
luisa.beghin@ uniroma1.it
Alessandro De Gregorio
alessandro.degregorio@ uniroma1.it
$1 \quad$ Department of Statistical Sciences, Sapienza, University of Rome, P. le Aldo Moro 5, Rome, Italy 
complexification of the spatial variable in the linear semigroup operator

$$
T_{t} f(x)=\frac{1}{\sqrt{2 \pi t}} \int_{-\infty}^{+\infty} f(x-y) e^{-\frac{y^{2}}{2 y}} d y
$$

representing the unique solution to the Cauchy problem

$$
\frac{\partial u}{\partial t}(x, t)=\frac{1}{2} \frac{\partial^{2} u}{\partial x^{2}}(x, t), \quad u(x, 0)=f(x),
$$

where $t>0, x \in \mathbb{R}$ and $f \in \mathrm{BUC}(\mathbb{R})$. This approach is interesting because, by exploiting the theory of semigroups of linear operators, it is possible to obtain a new complex version of the heat equation (see (2.2) below) and study the properties of its analytic solution (see [8,9]). Furthermore, it is worth to observe that in this framework a suitable probabilistic interpretation of the solution to (2.2) leads to a wrapped up Brownian motion on a circle. The stochastic analysis of complex diffusion equations still seems to be an unexplored research topic.

The above mentioned theory was developed starting from partial differential equations involving the standard time derivative. The aim of this paper is to study complex versions of the time-fractional heat equations obtained by complexifying the spatial variable only (and keeping the time variable real). The main idea is to complexify the spatial variable in the corresponding integral operator arising in the study of timefractional evolution equations. In particular, we study the stochastic solution of the complex heat equation, when the time-derivative is replaced by a convolution-type operator, which generalizes the Caputo fractional derivative. We will adopt the definition given in [6], i.e.

$$
\mathfrak{D}_{t}^{g} u(t):=\frac{d}{d t} \int_{0}^{t} w(t-s)(u(s)-u(0)) d s, \quad t \geq 0,
$$

where $w(\cdot)$ is the tail Lévy measure of a subordinator $\mathcal{H}_{g}(t), t \geq 0$ and $g(\cdot)$ is its Laplace exponent, i.e. $\mathbb{E} e^{-\theta \mathcal{H}_{g}(t)}=e^{-g(\theta) t}$, where $t, \theta \geq 0$ (see Section 3 for details on this definition). The so-called generalized fractional calculus has been developed in recent years, starting from Kochubei in [13], by many authors (see, among the others, $[10,14,23]$ ). They extend the traditional construct of fractional derivatives and integrals in order to allow a wider class of kernels. Indeed, it is immediate to check that, by choosing $w(t)=t^{-\alpha} / \Gamma(1-\alpha)$, for $\alpha \in(0,1)$, which coincides with the tail Lévy measure of an $\alpha$-stable subordinator $\mathcal{H}_{\alpha}(t)$, the fractional derivative in (1.1) reduces to the so-called "regularized Caputo derivative". Thus we will define the latter as

$$
\frac{\partial^{\alpha}}{\partial t^{\alpha}} u(t):=\frac{1}{\Gamma(1-\alpha)} \frac{d}{d t} \int_{0}^{t}(t-s)^{-\alpha}(u(s)-u(0)) d s, \quad t \geq 0 .
$$

It has been proved in [17] that the solution to

$$
\frac{\partial^{\alpha}}{\partial t^{\alpha}} u(x, t)=\Delta u(x, t), \quad t \geq 0, x \in \mathbb{R}^{d}, d \geq 1,
$$


with $u(x, 0)=f(x)$, admits the probabilistic representation $\mathbb{E}_{x}\left[f\left(B\left(\mathcal{E}_{\alpha}(t)\right)\right)\right]$, where $B:=\{B(t), t \geq 0\}$, is the standard Brownian motion on $\mathbb{R}^{d}$, with infinitesimal generator $\Delta$, and $\left\{\mathcal{E}_{\alpha}(t), t \geq 0\right\}$, is the inverse of the stable subordinator $\mathcal{H}_{\alpha}(t)$ (independent of $B$ ).

The previous result has been extended to the case of a strong Markov process $X:=\{X(t), t \geq 0\}$ (on a separable locally compact Hausdorff space $E$ ) whose transition semigroup is a uniformly bounded strong continuous semigroup in some Banach space and has infinitesimal generator $\mathcal{A}$. In this case, the solution to

$$
\left(\mathfrak{D}_{t}^{g}+b \frac{\partial}{\partial t}\right) u(x, t)=\mathcal{A} u(x, t), \quad t \geq 0, x \in E, b \geq 0,
$$

with $u(x, 0)=f(x)$, is represented by $\mathbb{E}_{x}\left[f\left(X\left(\mathcal{E}_{g}(t)\right)\right)\right]$, where $\mathcal{E}_{g}(t), t \geq 0$, is the inverse of the general subordinator $\mathcal{H}_{g}(t)$, with drift $b$, and is independent of $X$ (see [6], for details).

If we denote the unit disk as $D:=\{z \in \mathbb{C} ;|z|<1\}$ and we consider the space $A(D)=\{f: \bar{D} \rightarrow: f$ is analytic on $D$, continuous on $\bar{D}\}$, endowed with the uniform norm, then we study here the solution to the following Cauchy problem

$$
\left(\mathfrak{D}_{t}^{g}+b \frac{\partial}{\partial t}\right) u(z, t)=\frac{1}{2} \frac{\partial^{2} u}{\partial \varphi^{2}}(z, t), \quad(t, z) \in(0, \infty) \times D \backslash\{0\}, z=r e^{i \varphi},
$$

with $u(z, 0)=f(z)$, where $f(z) \in A(D), z \in \bar{D}$.

The paper is organized as follows. Section 2 contains the probabilistic interpretation of the solution to the complex Cauchy problem introduced in [8] and a discussion on the properties of the circular Brownian motion. The generalized fractional setting and the complex time-fractional heat equation are introduced in Section 3, where the stochastic solution related to the related complex Cauchy problem is obtained. Section 4 is devoted to the analysis of the time-changed Brownian motion emerging in the previous section. Some results on the moments of the process are provided, as well as the construction of the time-changed process based on the convergence of time-continuous random walks. Furthermore, some special cases involving stable and tempered stable subordinators are examined. The last section contains the analysis of the complex time-fractional heat equation in higher dimensions.

\section{On the probabilistic meaning of heat-type equations with complex space variables}

Let $D:=\{z \in \mathbb{C} ;|z|<1\}$ be the open unit disk and introduce the Banach space $(A(D),\|\cdot\|)$, where $A(D)=\{f: \bar{D} \rightarrow \mathbb{C}: f$ is analytic on $D$, continuous on $\bar{D}\}$, endowed with the uniform norm $\|f\|=\sup \{|f| ; z \in \bar{D}\}$. If $f \in A(D)$ then it can be represented in the series form $f(z)=\sum_{k=0}^{\infty} a_{k} z^{k}$. 
In the interesting paper [8], it was proved (see Theorem 2.1 in [8]) that the singular (at $t=0$ ) complex integral (that is known as a Gauss-Weierstrass integral)

$$
W_{t} f(z)=\frac{1}{\sqrt{2 \pi t}} \int_{-\infty}^{+\infty} f\left(z e^{-i u}\right) e^{-u^{2} / 2 t} d u, \quad t \geq 0
$$

is a $C_{0}$-contraction semigroup of linear operators on $A(D)$. Furthermore, $u(z, t)=$ $W_{t} f(z)$, is the unique solution (with $u(z, t) \in A(D)$ for a fixed $t$ ) for the Cauchy problem

$$
\frac{\partial u}{\partial t}(z, t)=\frac{1}{2} \frac{\partial^{2} u}{\partial \varphi^{2}}(z, t), \quad z=r e^{i \varphi}, 0<r<1, \varphi \in[0,2 \pi),
$$

under the initial condition

$$
u(z, 0)=f(z), \quad z \in \bar{D}
$$

where $f \in A(D)$.

Here we briefly discuss the interesting probabilistic meaning of this representation of the solution for the problem (2.2)-(2.3). Indeed, it is evident from (2.1), that the solution for the Cauchy problem can be represented as

$$
u(z, t)=\mathbb{E} f\left(z e^{-i B(t)}\right)=\mathbb{E} f\left(\mathfrak{B}_{z}(t)\right)
$$

where $B:=\{B(t), t \geq 0\}$ is the $\mathbb{R}$-valued Brownian motion on $(\Omega, \mathcal{F}, \mathbb{P})$ and $\mathfrak{B}_{z}(t)=z e^{-i B(t)}$. This means that the probabilistic representation of the solution for this complexified Cauchy problem is directly related to a circular or wrapped Brownian motion $\mathfrak{B}_{z}:=\left\{\mathfrak{B}_{z}(t), t \geq 0\right\}$ moving on a circle with radius $r \in(0,1]$ (hereafter denoted by $\mathbb{S}_{r}$ ) and starting point $z$. Furthermore, $\mathfrak{B}$ stands for $\mathfrak{B}_{1}$.

For the sake of simplicity, we set $z=1$. From the properties of the classical $\mathbb{R}$-valued Brownian motion, it is easy to characterized $\mathfrak{B}$. Let $\overline{\mathfrak{B}}$ be the complex conjugate of $\mathfrak{B}$. We observe that the wrapped Brownian motion $\mathfrak{B}$, satisfies the following properties:

1) $\mathfrak{B}(0)=1$ a.s.;

2) $\mathfrak{B}\left(t_{k}\right) \overline{\mathfrak{B}}\left(t_{k-1}\right)$ with $k=1,2, \ldots, n \in \mathbb{N}, 0=: t_{0} \leq t_{1}<t_{2}<\ldots<t_{n}<\infty$ are independent;

3) $\mathfrak{B}(t) \overline{\mathfrak{B}}(s)$ has the same distribution of $\mathfrak{B}(t+h) \overline{\mathfrak{B}}(s+h)$, where $0 \leq s<t, h \geq$ $-s$;

4) for $0 \leq s<t$,

$$
\mathfrak{B}(t) \overline{\mathfrak{B}}(s) \sim W N\left(0, e^{-\frac{t-s}{2}}\right),
$$


where $W N\left(\mu, e^{-\frac{\sigma^{2}}{2}}\right), \mu \in \mathbb{R}, \sigma^{2}>0$, stands for a wrapped normal random variables with probability density function given by

$$
f(\varphi)=\frac{1}{\sqrt{2 \pi} \sigma} \sum_{k=-\infty}^{\infty} e^{-\frac{(\varphi-\mu+2 k \pi)^{2}}{2 \sigma^{2}}}, \quad \varphi \in[0,2 \pi) .
$$

This result follows by standard arguments on the wrapped distributions; i.e. by wrapping the $N(0, t-s)$ onto the circle (see, e.g., [16]).

5) $\mathfrak{B}$ is a wrapped Gaussian process; i.e. let $0=: t_{0}<t_{1}<t_{2}<\ldots<t_{n}<\infty$, the random vector $\left(\mathfrak{B}\left(t_{1}\right), \mathfrak{B}\left(t_{2}\right), \ldots, \mathfrak{B}\left(t_{n}\right)\right)$ is multivariate wrapped normal in the following sense

$$
\prod_{k=1}^{n}\left(\mathfrak{B}\left(t_{k}\right)\right)^{\alpha_{k}}, \quad \alpha_{k} \in \mathbb{R}
$$

admits a one-dimensional wrapped gaussian distribution. Indeed,

$$
\prod_{k=1}^{n}\left(\mathfrak{B}\left(t_{k}\right)\right)^{\alpha_{k}}=e^{i \sum_{k=1}^{n} \alpha_{k} B\left(t_{k}\right)} .
$$

Since $B$ is a Gaussian process, it follows that $\sum_{k=1}^{n} \alpha_{k} B\left(t_{k}\right) \sim N\left(0, \sum_{k=1}^{n} \alpha_{k}^{2} t_{k}\right)$. Then, as in the previous point

$$
\prod_{k=1}^{n}\left(\mathfrak{B}\left(t_{k}\right)\right)^{\alpha_{k}} \sim W N\left(0, e^{-\frac{\sum_{k=1}^{n} \alpha_{k}^{2} t_{k}}{2}}\right) .
$$

\section{Time-fractional diffusive-type equations with a complex spatial variable}

Let us introduce a time-fractional version of the complex heat equation (2.2) and study its stochastic solution.

Let $g:(0,+\infty) \rightarrow \mathbb{R}$ be a Bernstein function (i.e. a non-negative, $C^{\infty}$ function such that $\left.(-1)^{k-1} g^{(k)}(x) \leq 0, \forall x>0, k \in \mathbb{N}\right)$. Then, it is well-known that the following representation holds (see e.g., [22])

$$
g(x)=a+b x+\int_{0}^{\infty}\left(1-e^{-s x}\right) \nu(d s), \quad b \geq 0,
$$

where $v(\cdot)$ is a non-negative measure on $(0,+\infty)$, satisfying the condition

$$
\int_{0}^{\infty}(z \wedge 1) v(d z)<\infty
$$


i.e. $v$ is a Lévy measure.

Let $w(s)=\int_{s}^{+\infty} v(d z)$ be its tail, in this paper we consider the following convolution-type derivative (see [6])

$$
\mathfrak{D}_{t}^{g} u(t):=\frac{d}{d t} \int_{0}^{t} w(t-s)(u(s)-u(0)) d s .
$$

Typically $w$ is a non-negative decreasing function on $(0,+\infty)$ that blows up at $x=0$ and locally integrable on $[0, \infty)$. We refer to [6] for the functional setting, observing that obviously this definition is a generalization of the Caputo fractional derivative (see, e.g., [12]): the latter is recovered, as a special case, for $w(s)=\frac{s^{-\alpha}}{\Gamma(1-\alpha)}$, with $\alpha \in(0,1)$. Observe that we used a quite different notation from [6] in order to underline the connection between this generalized fractional derivative and the particular choice of the underlying Bernstein function $g$. We remark that a similar probabilistic approach to the generalized time-fractional derivatives have been developed in [23]. It is similar but not equivalent. Hereafter, we exclude compound Poisson subordinator, namely we assume that $a=0$ and that the tail measure $w(\cdot)$ is infinite in the origin and absolutely continuous on $(0,+\infty)$. Let now $\mathcal{H}_{g}:=\left\{\mathcal{H}_{g}(t), t \geq 0\right\}$ be the subordinator with Lévy measure $v$ and Laplace exponent $g$, i.e.

$$
\mathbb{E}\left(e^{-\theta \mathcal{H}_{g}(t)}\right)=e^{-\operatorname{tg}(\theta)}, \quad \theta \geq 0
$$

(see, e.g., [1]). We denote by $\mathcal{E}_{g}:=\left\{\mathcal{E}_{g}(t), t \geq 0\right\}$, the inverse (or hitting-time) process $\mathcal{E}_{g}(t):=\inf \left\{s>0: \mathcal{H}_{g}(s)>t\right\}$, i.e.

$$
\left\{\mathcal{E}_{g}(t) \geq s\right\}=\left\{\mathcal{H}_{g}(s) \leq t\right\}, \quad \forall s, t \in \mathbb{R}^{+}
$$

By the assumptions on $w$, the subordinator $t \mapsto \mathcal{H}_{g}(t)$ associated to $g$ is strictly increasing a.s. As a consequence, its inverse $t \mapsto \mathcal{E}_{g}(t)$ is continuous a.s. We recall that the time-Laplace transform of the density of $\mathcal{E}_{g}(t)$ (with $t>0$ ) denoted by $m_{g}(s, t):=\mathbb{P}\left(\mathcal{E}_{g}(t) \in d s\right) / d s$ reads

$$
\int_{0}^{\infty} e^{-\theta t} m_{g}(s, t) d t=\frac{g(\theta)}{\theta} e^{-s g(\theta)}, \quad \theta \geq 0,
$$

see, for example, Proposition 3.2 in [23].

We now consider the standard $\mathbb{R}$-valued Brownian motion $B$, time-changed by $\mathcal{E}_{g}(t), t \geq 0$ (under the assumption that $B$ and $\mathcal{E}_{g}$ are mutually independent); i.e. $\left\{B\left(\mathcal{E}_{g}(t)\right), t \geq 0\right\}$. Then, the density of $B\left(\mathcal{E}_{g}(t)\right)$ for a fixed $t>0$, is given by

$$
\ell_{g}(x, t)=\int_{0}^{+\infty} \frac{e^{-\frac{x^{2}}{2 y}}}{\sqrt{2 \pi y}} m_{g}(y, t) d y, \quad x \in \mathbb{R} .
$$


Then, for all $t>0$, we can define on $D$, the following complex integral

$$
W_{t}^{g} f(z):=\int_{\mathbb{R}} f\left(z e^{-i u}\right) \ell_{g}(u, t) d u, \quad z \in \bar{D},
$$

where $\ell_{g}$ is given in (3.6). The following stochastic interpretation of (3.7) emerges

$$
W_{t}^{g} f(z)=\mathbb{E} f\left(\mathfrak{B}_{g}^{z}(t)\right),
$$

where

$$
\mathfrak{B}_{g}^{z}:=\left\{\mathfrak{B}_{g}^{z}(t), t \geq 0\right\}:=\left\{z e^{-i B\left(\mathcal{E}_{g}(t)\right)}, t \geq 0\right\},
$$

is the time-changed circular Brownian motion moving on a circle with radius $r \in(0,1]$ with starting point $z$, obtained from the wrapped up process $\mathfrak{B}^{z}$ introduced in the previous section. We observe that

$$
W_{t}^{g} f(z)=\mathbb{E}\left[W_{\mathcal{E}_{g}(t)} f(z)\right]=\int_{0}^{\infty} W_{y} f(z) m_{g}(y, t) d y,
$$

that is, $W_{t}^{g}$ arises by the time-changing of the $C_{0}$-semigroup (2.1).

We have the following analytic results concerning the convolution operator $W_{t}^{g}$.

Theorem 1 (i) If $f \in A(D)$, then we have that for any $t>0$, we have that

$$
W_{t}^{g}: A(D) \rightarrow A(D),
$$

i.e. $W_{t}^{g} f(z)$ is analytic in $D$

$$
W_{t}^{g} f(z)=\sum_{k=0}^{\infty} a_{k} z^{k} d_{k}(t)
$$

where $d_{k}(t):=\mathbb{E}\left[e^{-\frac{k^{2}}{2} \mathcal{E}_{g}(t)}\right]$, and if $f$ is continuous on $\bar{D}$, the integral $W_{t}^{g} f(z)$ is continuous on $\bar{D}$ as well. Furthermore,

$$
R_{\theta} W_{t}^{g} f(z):=\int_{0}^{\infty} e^{-\theta t} W_{t}^{g} f(z) d t=\sum_{k=0}^{\infty} a_{k} z^{k} \tilde{d}_{k}(\theta), \quad \theta \geq 0
$$

where

$$
\tilde{d}_{k}(\theta):=\frac{g(\theta) / \theta}{g(\theta)+\frac{k^{2}}{2}} .
$$

(ii) Moreover, $u(z, t)=W_{t}^{g} f(z)$ is the unique solution, belonging to $A(D)$ for any $t \geq 0$, of the Cauchy problem

$$
\left(\mathfrak{D}_{t}^{g}+b \frac{\partial}{\partial t}\right) u(z, t)=\frac{1}{2} \frac{\partial^{2} u}{\partial \varphi^{2}}(z, t),
$$




$$
\begin{gathered}
(t, z) \in(0, \infty) \times D \backslash\{0\}, z=r e^{i \varphi}, 0<r<1, \\
u(z, 0)=f(z), \quad f(z) \in A(D), z \in \bar{D} .
\end{gathered}
$$

Proof (i) The representation (3.10) follows by considering that $f(z) \in A(D)$ and by taking into account (3.6) together with (3.5). Indeed, since $f\left(z e^{-i u}\right)=$ $\sum_{k=0}^{\infty} a_{k} z^{k} e^{-i u k}, z \in D$, is absolutely convergent, we can write

$$
\begin{aligned}
W_{t}^{g} f(z) & =\sum_{k=0}^{\infty} a_{k} z^{k} \mathbb{E}\left[e^{-i k B\left(\mathcal{E}_{g}(t)\right)}\right] \\
& =\sum_{k=0}^{\infty} a_{k} z^{k} \int_{\mathbb{R}} e^{-i k u} \ell_{g}(u, t) \mathrm{d} u \\
& =\sum_{k=0}^{\infty} a_{k} z^{k} \int_{0}^{+\infty} \frac{1}{\sqrt{2 \pi y}} m_{g}(y, t) d y \int_{\mathbb{R}} e^{-i k u} e^{-\frac{u^{2}}{2 y}} \mathrm{~d} u \\
& =\sum_{k=0}^{\infty} a_{k} z^{k} \int_{0}^{+\infty} e^{-\frac{k^{2} y}{2}} m_{g}(y, t) d y \\
& =\sum_{k=0}^{\infty} a_{k} z^{k} \mathbb{E}\left(e^{-\frac{k^{2}}{2} \mathcal{E}_{g}(t)}\right) .
\end{aligned}
$$

By using the same arguments in [9], it is possible to prove that if $z_{n}, z_{0} \in \bar{D}$, with $\lim _{n \rightarrow \infty} z_{n}=z_{0}$, we get

$$
\begin{aligned}
\left|W_{t}^{g} f\left(z_{n}\right)-W_{t}^{g} f\left(z_{0}\right)\right| & \leq \int_{\mathbb{R}} \omega_{1}\left(f ;\left|z_{n}-z_{0}\right|\right) \bar{D} \ell_{g}(u, t) \mathrm{d} u \\
& =\omega_{1}\left(f ;\left|z_{n}-z_{0}\right|\right) \bar{D},
\end{aligned}
$$

where $\omega_{1}(f ; \delta)_{\bar{D}}:=\sup \{|f(u)-f(v)| ;|u-v| \leq \delta, u, v \in \bar{D}\}$ is the modulus of continuity of $f$. Then if $f$ is continuous on $\bar{D}$, the integral $W_{t}^{g} f(z)$ is continuous on $\bar{D}$, as $n \rightarrow \infty$.

From (3.10) and by exploiting (3.5), we obtain that

$$
\begin{aligned}
R_{\theta} W_{t}^{g} f(z) & =\sum_{k=0}^{\infty} a_{k} z^{k} \int_{0}^{\infty} e^{-\theta t} \mathbb{E}\left(e^{-\frac{k^{2}}{2} \mathcal{E}_{g}(t)}\right) d t \\
& =\sum_{k=0}^{\infty} a_{k} z^{k} \frac{g(\theta) / \theta}{\frac{k^{2}}{2}+g(\theta)}
\end{aligned}
$$

(ii) From Theorem 2.1 in [6], we can observe that: 1) $\mathfrak{D}_{t}^{g} W_{t}^{g} f(z)$ is well-defined since the integral appearing in the definition of $\mathfrak{D}_{t}^{g}$ is absolutely convergent in the Banach space $(A(D),\|\cdot\|)$; 2) for $b>0, t \mapsto W_{t}^{g} f(\cdot)$ is globally Lipschitz in $(A(D),\|\cdot\|)$ and then $\frac{\partial}{\partial t} W_{t}^{g} f(\cdot)$ exits in $(A(D),\|\cdot\|)$ for a.s. $t \geq 0$. In order to 
prove that (3.10) coincides with the solution of (3.12) with initial condition (3.13) we take the time-Laplace transform of both sides of (3.12). By applying the result (2.18) in [21], on the Laplace transform of the generalized derivative (3.2), we obtain

$$
\begin{aligned}
\mathcal{L}\left\{\mathfrak{D}_{t}^{g} W_{t}^{g} f(z)+b \frac{\partial}{\partial t} W_{t}^{g} f(z) ; \theta\right\} & =g(\theta) R_{\theta} W_{t}^{g} f(z)-\frac{g(\theta)}{\theta} f(z) \\
& =g(\theta) \sum_{k=0}^{\infty} a_{k} z^{k} \tilde{d}_{k}(\theta)-\frac{g(\theta)}{\theta} f(z) \\
& =\frac{g(\theta)}{\theta}\left[\sum_{k=0}^{\infty} \frac{a_{k} z^{k} g(\theta)}{g(\theta)+\frac{k^{2}}{2}}-f(z)\right]
\end{aligned}
$$

and

$$
\begin{aligned}
\frac{1}{2} \frac{\partial^{2}}{\partial \varphi^{2}} \mathcal{L}\left\{W_{t}^{g} f(z) ; \theta\right\} & =\frac{1}{2} \frac{\partial^{2}}{\partial \varphi^{2}} \sum_{k=0}^{\infty} a_{k} z^{k} \tilde{d}_{k}(\theta) \\
& =-\frac{1}{2} \frac{g(\theta)}{\theta} \sum_{k=0}^{\infty} \frac{a_{k} k^{2} z^{k}}{\frac{k^{2}}{2}+g(\theta)}
\end{aligned}
$$

Furthermore, from (3.10), it is easy to prove that

$$
W_{0}^{g} f(z)=f(z)
$$

The result follows by considering (3.14), (3.15) and (3.16) together and taking into account that $f(z)=\sum_{k=0}^{\infty} a_{k} z^{k}$.

Remark 1 In Theorem 1, we proved that $u(t, z)=W_{t}^{g} f(z)$ is the classical solution with $u \in C^{\infty}([0, \infty) ; A(D))$. Actually, it is also possible to prove that $u(t, z)$ is the unique strong solution of the fractional Cauchy problem (3.12)-(3.13) (see Theorem 2.1 in [6] for the exact statement).

\section{Time-changed wrapped Brownian motion}

"Let $z=1$ ", by exploiting the theory of wrapped distribution, for any $t>0$ we can write down the probability density of $\mathfrak{B}_{g}(t):=e^{i B\left(\mathcal{E}_{g}(t)\right)}, t \geq 0$,

$$
\mu_{\mathfrak{B}_{g}}(\varphi, t):=\frac{\mathbb{P}\left(\mathfrak{B}_{g}(t) \in d \varphi\right)}{d \varphi}=\sum_{k=-\infty}^{\infty} \ell_{g}(\varphi+2 k \pi, t), \quad \varphi \in[0,2 \pi)
$$


The probability distribution on a circle is characterized by its Fourier coefficients (see [7], Theorem XIX 6.1). In our case, for $k \in \mathbb{N}$, we have that

$$
\begin{aligned}
\phi_{k}^{t} & :=\int_{0}^{2 \pi} e^{i k \varphi} \mu_{\mathfrak{B}_{g}}(\varphi, t) d \varphi=\sum_{k=-\infty}^{\infty} \int_{2 k \pi}^{2 \pi(k+1)} e^{i k \varphi} \ell_{g}(\varphi, t) d \varphi \\
& =\mathbb{E}\left[e^{i k B\left(\mathcal{E}_{g}(t)\right)}\right]=\mathbb{E}\left[e^{-\frac{k^{2} \mathcal{E}_{g}(t)}{2}}\right] \\
& =d_{k}(t) .
\end{aligned}
$$

Therefore,

$$
\begin{aligned}
\mu_{\mathfrak{B}_{g}}(\varphi) & =\frac{1}{2 \pi} \sum_{k=-\infty}^{\infty} \phi_{k}^{t} e^{-i k \varphi} \\
& =\frac{1}{2 \pi}\left(1+2 \sum_{k=1}^{\infty} d_{k}(t) \cos (k \varphi)\right) .
\end{aligned}
$$

We now evaluate the Laplace transform of the first moments of $\mathfrak{B}_{g}(t)$ by applying the results on the joint moments of the inverse subordinators given in [24]. In particular, we recall that, for the Laplace transform of $K_{t_{1}, \ldots, t_{n}}\left(s_{1}, \ldots, s_{n}\right):=$ $\mathbb{P}\left(\mathcal{E}_{g}\left(t_{1}\right)>s_{1}, \ldots, \mathcal{E}_{g}\left(t_{n}\right)>s_{n}\right)$, the following formula holds

$$
\begin{aligned}
\widetilde{K}_{\theta_{1}, \ldots, \theta_{n}}\left(s_{1}, \ldots, s_{n}\right) & :=\int_{0}^{\infty} \ldots \int_{0}^{\infty} e^{-\theta_{1} t_{1} \ldots-\theta_{n} t_{n}} K_{t_{1}, \ldots, t_{n}}\left(s_{1}, \ldots, s_{n}\right) d t_{1} \ldots d t_{n} \\
& =\frac{1}{\prod_{j=1}^{n} \theta_{j}} \exp \left\{-\sum_{j=1}^{n} g\left(\sum_{k=j}^{n} \theta_{j(n)}\right)\left(s_{j(i)}-s_{j(i-1)}\right)\right\},
\end{aligned}
$$

where $\theta_{1}, \ldots, \theta_{n}>0,0=s_{j(0)} \leq s_{j(1)} \leq \ldots \leq s_{j(n)}$ and $j(1), \ldots, j(n)$ is a permutation of the integers $1, \ldots, n$ (by convention $j(0)=0$ ).

Theorem 2 The Laplace transform of the moments of the process $\mathfrak{B}_{g}$ is equal to

$$
\mathcal{L}\left\{\mathbb{E}\left[\mathfrak{B}_{g}(t)\right]^{r} ; \theta\right\}=\frac{2 g(\theta)}{\theta(r+2 g(\theta))}, \quad \theta>0, t \geq 0, r \in \mathbb{N}
$$

and

$$
\begin{aligned}
\mathcal{L} & \left\{\mathbb{E}\left(\mathfrak{B}_{g}\left(t_{1}\right) \mathfrak{B}_{g}\left(t_{2}\right)\right) ; \theta_{1}, \theta_{2}\right\} \\
& =\frac{4 g\left(\theta_{1}\right) g\left(\theta_{2}\right)\left[g\left(\theta_{1}+\theta_{2}\right)+2\right]+3\left[g\left(\theta_{1}\right)+g\left(\theta_{2}\right)-g\left(\theta_{1}+\theta_{2}\right)\right]}{\theta_{1} \theta_{2}\left[2+g\left(\theta_{1}+\theta_{2}\right)\right]\left(3+2 g\left(\theta_{1}\right)\right)\left(1+2 g\left(\theta_{2}\right)\right)},
\end{aligned}
$$

for $0 \leq t_{1}<t_{2}$ and $\theta_{1}, \theta_{2}>0$. 
Proof The $r$-th moment in (4.2) can be easily obtained by a conditioning argument and by considering (3.3):

$$
\begin{aligned}
V(t) & :=\mathbb{E}\left[e^{i B\left(\mathcal{E}_{g}(t)\right)}\right]^{r}=\mathbb{E}\left[\mathbb{E}\left[e^{i r B\left(\mathcal{E}_{g}(t)\right)} \mid \mathcal{E}_{g}(t)\right]\right] \\
& =\mathbb{E} e^{-\frac{r}{2} \mathcal{E}_{g}(t)}=\int_{0}^{+\infty} e^{-\frac{r s}{2}} m_{g}(s, t) d s .
\end{aligned}
$$

Then, by taking the time-Laplace transform and considering (4.5), we have that, for $\theta>0$,

$$
\widetilde{V}(\theta)=\int_{0}^{+\infty} e^{-\theta t} V(t) d t=\int_{0}^{+\infty} e^{-\frac{r s}{2}} \tilde{m}_{g}(s, \theta) d s=\frac{g(\theta)}{\theta(r / 2+g(\theta))}
$$

where $\tilde{m}_{g}(s, \theta):=\int_{0}^{+\infty} e^{-\theta t} m_{g}(s, t) d t$. In order to prove formula (4.3), we write

$$
\begin{aligned}
V\left(t_{1}, t_{2}\right) & :=\mathbb{E}\left[\mathfrak{B}_{g}\left(t_{1}\right) \mathfrak{B}_{g}\left(t_{2}\right)\right] \\
& =\mathbb{E} e^{i B\left(\mathcal{E}_{g}\left(t_{1}\right)\right)+i B\left(\mathcal{E}_{g}\left(t_{2}\right)\right)}=\mathbb{E}\left[\mathbb{E}\left[e^{i B\left(\mathcal{E}_{g}\left(t_{1}\right)\right)+i B\left(\mathcal{E}_{g}\left(t_{2}\right)\right)} \mid \mathcal{E}_{g}\left(t_{1}\right), \mathcal{E}_{g}\left(t_{1}\right)\right]\right] \\
& =\mathbb{E} e^{-\frac{1}{2}\left[\mathcal{E}_{g}\left(t_{1}\right)+\mathcal{E}_{g}\left(t_{2}\right)+2 \min \left\{\mathcal{E}_{g}\left(t_{1}\right), \mathcal{E}_{g}\left(t_{2}\right)\right\}\right]}
\end{aligned}
$$

We start by evaluating, for any $\eta_{1}, \eta_{2}>0$

$$
\begin{aligned}
V_{\eta_{1}, \eta_{2}}\left(t_{1}, t_{2}\right)= & \mathbb{E} e^{-\eta_{1} \mathcal{E}_{g}\left(t_{1}\right)-\eta_{2} \mathcal{E}_{g}\left(t_{2}\right)} \\
= & \int_{0}^{+\infty} \int_{0}^{+\infty} e^{-\eta_{1} s_{1}-\eta_{2} s_{2}} \frac{\partial^{2}}{\partial s_{1} \partial s_{2}} K_{t_{1}, t_{2}}\left(s_{1}, s_{2}\right) d s_{1} d s_{2} \\
= & {[\text { by repeatedly integrating by parts }] } \\
= & K_{t_{1}, t_{2}}(0,0)-\eta_{1} \int_{0}^{+\infty} e^{-\eta_{1} s_{1}} K_{t_{1}, t_{2}}\left(s_{1}, 0\right) d s_{1} \\
& -\eta_{2} \int_{0}^{+\infty} e^{-\eta_{2} s_{2}} K_{t_{1}, t_{2}}\left(0, s_{2}\right) d s_{2} \\
& +\eta_{1} \eta_{2} \int_{0}^{+\infty} \int_{0}^{+\infty} e^{-\eta_{1} s_{1}-\eta_{2} s_{2}} K_{t_{1}, t_{2}}\left(s_{1}, s_{2}\right) d s_{1} d s_{2} .
\end{aligned}
$$

We now consider that $K_{t_{1}, t_{2}}(0,0)=\mathbb{P}\left(\mathcal{E}_{g}\left(t_{1}\right)>0, \mathcal{E}_{g}\left(t_{2}\right)>0\right)=1$ and that $K_{t_{1}, t_{2}}\left(s_{1}, 0\right)=\mathbb{P}\left(\mathcal{E}_{g}\left(t_{1}\right)>s_{1}\right)$, so that we can write

$$
\begin{aligned}
\int_{0}^{+\infty} e^{-\eta s_{1}} K_{t_{1}, t_{2}}\left(s_{1}, 0\right) d s_{1} & =\int_{0}^{+\infty} e^{-\eta s_{1}} \mathbb{P}\left(\mathcal{E}_{g}\left(t_{1}\right)>s_{1}\right) d s_{1} \\
& =\frac{1}{\eta}\left[1-\tilde{m}_{g}\left(\eta, t_{1}\right)\right]
\end{aligned}
$$


and analogously for $K_{t_{1}, t_{2}}\left(0, s_{2}\right)$. Therefore, we get

$$
\begin{aligned}
V_{\eta_{1}, \eta_{2}}\left(t_{1}, t_{2}\right)= & \tilde{m}_{g}\left(\eta_{1}, t_{1}\right)+\tilde{m}_{g}\left(\eta_{2}, t_{2}\right)-1 \\
& +\eta_{1} \eta_{2} \int_{0}^{+\infty} \int_{0}^{+\infty} e^{-\eta_{1} s_{1}-\eta_{2} s_{2}} K_{t_{1}, t_{2}}\left(s_{1}, s_{2}\right) d s_{1} d s_{2}
\end{aligned}
$$

In order to apply (4.1), we evaluate the Laplace transform of (4.5), with respect to the time variables:

$$
\begin{aligned}
\widetilde{V}_{\eta_{1}, \eta_{2}}\left(\theta_{1}, \theta_{2}\right): & =\int_{0}^{+\infty} \int_{0}^{+\infty} e^{-\theta_{1} t_{1}-\theta_{2} t_{2}} V_{\eta_{1}, \eta_{2}}\left(t_{1}, t_{2}\right) d t_{1} d t_{2} \\
= & \frac{1}{\theta_{2}} \widetilde{\widetilde{m}}_{g}\left(\eta_{1}, \theta_{1}\right)+\frac{1}{\theta_{1}} \widetilde{\widetilde{m}}_{g}\left(\eta_{2}, \theta_{2}\right)-\frac{1}{\theta_{1} \theta_{2}} \\
& +\eta_{1} \eta_{2} \int_{0}^{+\infty} \int_{0}^{+\infty} e^{-\eta_{1} s_{1}-\eta_{2} s_{2}} \widetilde{K}_{\theta_{1}, \theta_{2}}\left(s_{1}, s_{2}\right) d s_{1} d s_{2},
\end{aligned}
$$

where $\widetilde{\widetilde{m}}_{g}(\eta, \theta):=\int_{0}^{+\infty} e^{-\eta s} \tilde{m}_{g}(s, \theta) d s$. On the other hand, we can rewrite the last integral in (4.6), by applying (4.1), as follows

$$
\begin{aligned}
& \frac{1}{\theta_{1} \theta_{2}} \int_{0}^{+\infty} \int_{s_{1}}^{+\infty} e^{-\eta_{1} s_{1}-\eta_{2} s_{2}} e^{-s_{1}\left[g\left(\theta_{1}+\theta_{2}\right)-g\left(\theta_{2}\right)\right]-s_{2} g\left(\theta_{2}\right)} d s_{1} d s_{2} \\
& +\frac{1}{\theta_{1} \theta_{2}} \int_{0}^{+\infty} \int_{0}^{s_{1}} e^{-\eta_{1} s_{1}-\eta_{2} s_{2}} e^{-s_{2}\left[g\left(\theta_{1}+\theta_{2}\right)-g\left(\theta_{1}\right)\right]-s_{1} g\left(\theta_{1}\right)} d s_{1} d s_{2} \\
& =\frac{1}{\theta_{1} \theta_{2}} \int_{0}^{+\infty} \int_{s_{1}}^{+\infty} e^{-\left[\eta_{1}+g\left(\theta_{1}+\theta_{2}\right)-g\left(\theta_{2}\right)\right] s_{1}-\left[\eta_{2}+g\left(\theta_{2}\right)\right] s_{2}} d s_{1} d s_{2} \\
& +\frac{1}{\theta_{1} \theta_{2}} \int_{0}^{+\infty} \int_{s_{2}}^{+\infty} e^{-\left[\eta_{1}+g\left(\theta_{1}\right)\right] s_{1}-\left[\eta_{2}+g\left(\theta_{1}+\theta_{2}\right)-g\left(\theta_{1}\right)\right] s_{2}} d s_{1} d s_{2} \\
& =\frac{1}{\theta_{1} \theta_{2}}\left[\int_{0}^{+\infty} \int_{s_{1}}^{+\infty} e^{-s_{1} A_{1}-s_{2} B_{2}} d s_{1} d s_{2}+\int_{0}^{+\infty} \int_{s_{2}}^{+\infty} e^{-s_{1} B_{1}-s_{2} A_{2}} d s_{1} d s_{2}\right] \\
& =\frac{1}{\theta_{1} \theta_{2}}\left[\frac{1}{B_{2}\left(A_{1}+B_{2}\right)}+\frac{1}{B_{1}\left(A_{2}+B_{1}\right)}\right]
\end{aligned}
$$

where we put $A_{i}:=\eta_{i}+g\left(\theta_{1}+\theta_{2}\right)-g\left(\theta_{j}\right)$, for $i, j=1,2$ and $i \neq j, B_{i}:=\eta_{i}+g\left(\theta_{i}\right)$, for $i=1,2$. We consider that $A_{1}+B_{2}=A_{2}+B_{1}=\eta_{1}+\eta_{2}+g\left(\theta_{1}+\theta_{2}\right)$, so that we can write (4.6), by recalling (4.5), as

$$
\begin{aligned}
\widetilde{V}_{\eta_{1}, \eta_{2}}\left(\theta_{1}, \theta_{2}\right)= & \frac{1}{\theta_{1} \theta_{2}}\left[\frac{g\left(\theta_{1}\right)}{\eta_{1}+g\left(\theta_{1}\right)}+\frac{g\left(\theta_{2}\right)}{\eta_{2}+g\left(\theta_{2}\right)}-1\right] \\
& +\frac{\eta_{1} \eta_{2}}{\theta_{1} \theta_{2}} \frac{\eta_{1}+\eta_{2}+g\left(\theta_{1}\right)+g\left(\theta_{2}\right)}{\left[\eta_{1}+\eta_{2}+g\left(\theta_{1}+\theta_{2}\right)\right]\left[\eta_{1}+g\left(\theta_{1}\right)\right]\left[\eta_{2}+g\left(\theta_{2}\right)\right]} .
\end{aligned}
$$


By taking into account that $\mathcal{H}_{g}$ is a.s. increasing and its inverse $\mathcal{E}_{g}$ is a.s. nondecreasing, so that $\min \left\{\mathcal{E}_{g}\left(t_{1}\right), \mathcal{E}_{g}\left(t_{2}\right)\right\}=\mathcal{E}_{g}\left(t_{1}\right)$ a.s., for $t_{2}>t_{1}$, formula (4.3) follows from (4.7), with $\eta_{1}=3 / 2$ and $\eta_{2}=1 / 2$, after some algebraic calculations.

We are also able to give an integral representation for the mixed moment $\mathbb{E}\left[\mathfrak{B}_{g}(t) \overline{\mathfrak{B}_{g}(s)}\right]$, for $t, s \geq 0$.

Theorem 3 Let $U_{g}(\tau):=\mathbb{E}\left[\mathcal{E}_{g}(\tau)\right], \tau \geq 0$ and $\mathbb{E}\left[\left(\mathcal{E}_{g}(t)\right)^{k}\right]<\infty, k \in \mathbb{N}$, then

$$
\mathbb{E}\left[\mathfrak{B}_{g}(t) \overline{\mathfrak{B}_{g}(s)}\right]=\mathbb{E} \mathfrak{B}_{g}(t \vee s)+\frac{1}{2} \int_{0}^{t \wedge s} \mathbb{E} \mathfrak{B}_{g}(t \vee s-\tau) d U_{g}(\tau)
$$

for $t, s \geq 0$ and $t \neq s$, while $\mathbb{E}\left[\mathfrak{B}_{g}(t) \overline{\mathfrak{B}_{g}(t)}\right]=1$.

Proof Let $t>s$ and denoting $U_{g}(s, t ; k, j):=\mathbb{E}\left[\left(\mathcal{E}_{g}(s)\right)^{k}\left(\mathcal{E}_{g}(t)\right)^{j}\right], s, t \geq 0, k, j \in$ $\mathbb{N}$, we can write down

$$
\begin{aligned}
\mathbb{E}\left[\mathfrak{B}_{g}(t) \overline{\mathfrak{B}_{g}(s)}\right]= & \mathbb{E}\left[e^{i\left(B\left(\mathcal{E}_{g}(t)\right)-B\left(\mathcal{E}_{g}(s)\right)\right)}\right] \\
= & \mathbb{E}\left[\left[e^{i\left(B\left(\mathcal{E}_{g}(t)\right)-B\left(\mathcal{E}_{g}(s)\right)\right)} \mid \mathcal{E}_{g}(t), \mathcal{E}_{g}(s)\right]\right] \\
= & \mathbb{E}\left[e^{-\frac{1}{2}\left(\mathcal{E}_{g}(t)-\mathcal{E}_{g}(s)\right)}\right] \\
= & \sum_{k=0}^{\infty} \frac{1}{k !}\left(-\frac{1}{2}\right)^{k} \mathbb{E}\left[\left(\mathcal{E}_{g}(t)-\mathcal{E}_{g}(s)\right)^{k}\right] \\
= & \sum_{k=0}^{\infty} \frac{1}{k !}\left(-\frac{1}{2}\right)^{k} \sum_{j=0}^{k}(-1)^{k-j}\left(\begin{array}{c}
k \\
j
\end{array}\right) \mathbb{E}\left[\left(\mathcal{E}_{g}(t)\right)^{j}\left(\mathcal{E}_{g}(s)\right)^{k-j}\right] \\
= & \sum_{k=1}^{\infty} \frac{1}{k !}\left(-\frac{1}{2}\right)^{k} \sum_{j=0}^{k-1}(-1)^{k-j}\left(\begin{array}{c}
k \\
j
\end{array}\right) U_{g}(s, t ; k-j, j) \\
& +\sum_{k=0}^{\infty} \frac{1}{k !}\left(-\frac{1}{2}\right)^{k} \mathbb{E}\left[\left(\mathcal{E}_{g}(t)\right)^{k}\right] \\
= & \sum_{k=1}^{\infty} \frac{1}{k !}\left(-\frac{1}{2}\right)^{k} \sum_{j=0}^{k-1}(-1)^{k-j}\left(\begin{array}{l}
k \\
j
\end{array}\right) U_{g}(s, t ; k-j, j)+\mathbb{E} e^{-\frac{1}{2} \mathcal{E}_{g}(t)},
\end{aligned}
$$

where we have singled out the term $j=k$ in the second summation, since it must be treated separately (in view of its different behavior for $s=0$ ). Now we use the recursive representation of the moments given by Theorem 4.2, [24], so that (4.9) can be rewritten as follows

$$
\mathbb{E}\left[\mathfrak{B}_{g}(t) \overline{\mathfrak{B}_{g}(s)}\right]=\sum_{k=1}^{\infty} \frac{1}{k !}\left(-\frac{1}{2}\right)^{k} \sum_{j=0}^{k-1}(-1)^{k-j}\left(\begin{array}{l}
k \\
j
\end{array}\right)
$$




$$
\begin{aligned}
& \times \int_{0}^{s}(k-j) U_{g}(s-\tau, t-\tau ; k-j-1, j) d U_{g}(\tau) \\
& +\sum_{k=1}^{\infty} \frac{1}{k !}\left(-\frac{1}{2}\right)^{k} \sum_{j=0}^{k-1}(-1)^{k-j}\left(\begin{array}{l}
k \\
j
\end{array}\right) \\
& \times \int_{0}^{s} j U_{g}(s-\tau, t-\tau ; k-j, j-1) d U_{g}(\tau)+\mathbb{E} \mathfrak{B}_{g}(t) \\
= & : I_{1}+I_{2}+\mathbb{E} \mathfrak{B}_{g}(t) .
\end{aligned}
$$

The first term in (4.10) can be treated as follows

$$
\begin{aligned}
I_{1}= & \sum_{k=1}^{\infty} \frac{1}{k !}\left(-\frac{1}{2}\right)^{k} \sum_{j=0}^{k-1}(-1)^{k-j}\left(\begin{array}{c}
k \\
j
\end{array}\right) \\
& \times \int_{0}^{s}(k-j) U_{g}(s-\tau, t-\tau ; k-j-1, j) d U_{g}(\tau) \\
= & \sum_{k=1}^{\infty} \frac{1}{(k-1) !}\left(-\frac{1}{2}\right)^{k} \sum_{j=0}^{k-1}(-1)^{k-j}\left(\begin{array}{c}
k-1 \\
j
\end{array}\right) \\
& \times \int_{0}^{s} \mathbb{E}\left[\left(\mathcal{E}_{g}(t-\tau)\right)^{j}\left(\mathcal{E}_{g}(s-\tau)\right)^{k-1-j}\right] d U_{g}(\tau) \\
= & \frac{1}{2} \sum_{l=0}^{\infty} \frac{1}{l !}\left(-\frac{1}{2}\right)^{l} \sum_{j=0}^{l}(-1)^{l-j}\left(\begin{array}{l}
l \\
j
\end{array}\right) \int_{0}^{s} \mathbb{E}\left[\left(\mathcal{E}_{g}(t-\tau)\right)^{j}\left(\mathcal{E}_{g}(s-\tau)\right)^{l-j}\right] d U_{g}(\tau) \\
= & \frac{1}{2} \int_{0}^{s} \mathbb{E}\left[\mathfrak{B}_{g}(t-\tau) \overline{\mathfrak{B}_{g}(s-\tau)}\right] d U_{g}(\tau),
\end{aligned}
$$

while the second one reads

$$
\begin{aligned}
I_{2}= & \sum_{k=1}^{\infty} \frac{1}{k !}\left(-\frac{1}{2}\right)^{k} \sum_{j=0}^{k-1}(-1)^{k-j}\left(\begin{array}{l}
k \\
j
\end{array}\right) \\
& \times \int_{0}^{s} j U_{g}(s-\tau, t-\tau ; k-j, j-1) d U_{g}(\tau) \\
= & \sum_{k=2}^{\infty} \frac{1}{(k-1) !}\left(-\frac{1}{2}\right)^{k} \sum_{j=1}^{k-1}(-1)^{k-j}\left(\begin{array}{c}
k-1 \\
j-1
\end{array}\right) \\
& \times \int_{0}^{s} \mathbb{E}\left[\left(\mathcal{E}_{g}(t-\tau)\right)^{j-1}\left(\mathcal{E}_{g}(s-\tau)\right)^{k-j}\right] d U_{g}(\tau) \\
= & \sum_{k=2}^{\infty} \frac{1}{(k-1) !}\left(-\frac{1}{2}\right)^{k} \sum_{m=0}^{k-2}(-1)^{k-m-1}\left(\begin{array}{c}
k-1 \\
m
\end{array}\right) \\
& \times \int_{0}^{s} \mathbb{E}\left[\left(\mathcal{E}_{g}(t-\tau)\right)^{m}\left(\mathcal{E}_{g}(s-\tau)\right)^{k-m-1}\right] d U_{g}(\tau)
\end{aligned}
$$




$$
\begin{aligned}
= & -\frac{1}{2} \sum_{k=2}^{\infty} \frac{1}{(k-1) !}\left(-\frac{1}{2}\right)^{k-1} \int_{0}^{s} \mathbb{E}\left[\left(\mathcal{E}_{g}(t-\tau)-\mathcal{E}_{g}(s-\tau)\right)^{k-1}\right] d U_{g}(\tau) \\
& +\frac{1}{2} \sum_{k=2}^{\infty} \frac{1}{(k-1) !}\left(-\frac{1}{2}\right)^{k-1} \int_{0}^{s} \mathbb{E}\left[\left(\mathcal{E}_{g}(t-\tau)\right)^{k-1}\right] d U_{g}(\tau) \\
= & -\frac{1}{2} \int_{0}^{s} \mathbb{E}\left[\mathfrak{B}_{g}(t-\tau) \overline{\mathfrak{B}_{g}(s-\tau)}\right] d U_{g}(\tau)+\frac{1}{2} \int_{0}^{s} \mathbb{E} \mathfrak{B}_{g}(t-\tau) d U_{g}(\tau) .
\end{aligned}
$$

The result follows by inserting $I_{1}$ and $I_{2}$ into (4.10) and treating the case $s>t$ analogously.

We now prove that the wrapped Brownian motion $\mathfrak{B}_{g}(t)$ can be obtained as a scaling limit of a transformed continuous-time random walk on a circle. Let us denote by $\stackrel{J_{1}}{\Longrightarrow}$ the convergence in the $J_{1}$ topology and by $\stackrel{M_{1}}{\Longrightarrow}$ the convergence in the $M_{1}$ topology in the Skorohod space $D\left([0, T), \mathbb{R}^{d}\right)$, for $T>0$ and $d=1,2, \ldots$ (see [25] and [19] for details on $J_{1}$ and $M_{1}$ topologies).

Theorem 4 Let $c>0$ and let $Y_{j}^{(c)}, j=1,2, \ldots$, be i.i.d. random variables with finite moments and scale parameter c. Let moreover $J_{j}^{(c)}, j=1,2, \ldots$, be i.i.d. random variables, independent of $Y_{j}^{(c)}$, for any $j=1,2, \ldots$, and for any $c>0$ and such that $\left\{T^{(c)}(c t), t \geq 0\right\}:=\left\{\sum_{j=1}^{[c t]} J_{j}^{(c)}, t \geq 0\right\} \stackrel{J_{1}}{\Rightarrow}\left\{\mathcal{H}_{g}(t), t \geq 0\right\}$ as $c \rightarrow+\infty$, in $D\left([0,+\infty), \mathbb{R}^{+}\right)$. Then

$$
\left\{e^{i \sum_{j=1}^{N_{t}^{(c)}} Y_{j}^{(c)}}, t \geq 0\right\} \stackrel{M_{1}}{\Longrightarrow}\left\{\mathfrak{B}_{g}(t), t \geq 0\right\}, \quad c \rightarrow+\infty
$$

in $D\left([0,+\infty), \mathbb{S}_{1}\right)$, where $N_{t}^{(c)}:=\max \left\{n \geq 0: T^{(c)}(n) \leq t\right\}$.

Proof The convergence in (4.11) follows by the application of Theorem 2.1 and Corollary 2.4 in [18], in the special case where $A(t)=B(t), t \geq 0$ : indeed, let $\operatorname{Disc}(x)$ be the set of discontinuities of $x$, the assumption that Disc $\left(\{A(t)\}_{t \geq 0}\right) \cap \operatorname{Disc}$ $\left(\left\{\mathcal{H}_{g}(t)\right\}_{t \geq 0}\right)=\emptyset$ a.s. is automatically satisfied because, as well-known, it is always possible to choose a version of Brownian motion such that its trajectories are continuous with probability one. Moreover, the Lévy measure of $\mathcal{H}_{g}$ is infinite on $[0,+\infty)$ by assumption. By the independence of $J_{j}^{(c)}$ and $Y_{j}^{(c)}$, for any $j=1,2 \ldots$ and by the functional central limit theorem, we have that

$$
\left\{\sum_{j=1}^{[c t]} Y_{j}^{(c)}, T^{(c)}(c t), t \geq 0\right\} \stackrel{J_{1}}{\Rightarrow}\left\{B(t), \mathcal{H}_{g}(t), t \geq 0\right\}, \quad c \rightarrow+\infty
$$


in the $J_{1}$ topology on $D\left([0,+\infty), \mathbb{R} \times \mathbb{R}^{+}\right)$. Therefore, by the above mentioned Theorem 2.1 in [18], the following convergence holds

$$
\left\{\sum_{j=1}^{N_{t}^{(c)}} Y_{j}^{(c)}, t \geq 0\right\} \stackrel{M_{1}}{\Rightarrow}\left\{B\left(\mathcal{E}_{g}(t)\right), t \geq 0\right\}, \quad c \rightarrow+\infty,
$$

in the $M_{1}$ topology on $D([0,+\infty), \mathbb{R})$. The result finally follows by applying the continuous mapping theorem to the function $\phi(\cdot): \mathbb{R} \rightarrow \mathbb{C}$ defined as $\phi(x)=e^{i x}$, $x \in \mathbb{R}$.

\subsection{The stable case}

For $g(\theta)=\theta^{\alpha}, \alpha \in(0,1)$, formula (3.1) holds for $a=0$ and $b=\lim _{\theta \rightarrow+\infty} g(\theta) / \theta$ $=0$. Moreover, the process $\mathcal{E}_{g}(t)$ reduces to the inverse of the $\alpha$-stable subordinator (see e.g. [20]) and the operator $\mathfrak{D}_{t}^{g}$ coincides with the Caputo time-fractional derivative of order $\alpha$, namely $\partial^{\alpha} / \partial t^{\alpha}$. In this case, if we denote $W_{t}^{g}$ as $W_{t}^{\alpha}$, we have that

$$
W_{t}^{\alpha}(f)(z)=\frac{1}{t^{\alpha / 2}} \int_{\mathbb{R}} f\left(z e^{i u}\right) W_{-\frac{\alpha}{2}, 1-\frac{\alpha}{2}}\left(-\frac{|u|}{t^{\alpha / 2}}\right) d u
$$

where

$$
W_{\beta, \gamma}(x)=\sum_{k=0}^{\infty} \frac{x^{k}}{k ! \Gamma(\beta k+\gamma)},
$$

is the Wright function, defined for $\beta, \gamma, x \in \mathbb{C}$. The representation (4.12) follows by the fact that the fundamental solution of the time-fractional diffusion equation

$$
\frac{\partial^{\alpha}}{\partial t^{\alpha}} u(x, t)=\frac{1}{2} \frac{\partial^{2}}{\partial x^{2}} u(x, t)
$$

involving Caputo time-fractional derivatives of order $\alpha \in(0,1)$ is given by

$$
u(x, t)=\frac{1}{t^{\alpha / 2}} W_{-\alpha / 2,1-\alpha / 2}\left(-\frac{|x|}{t^{\alpha / 2}}\right),
$$

see for example [15].

Then, in this case, as a consequence of Theorem 1, we have the following result.

Corollary 1 Let $E_{\alpha}(x):=\sum_{j=0}^{\infty} \frac{x^{j}}{\Gamma(\alpha j+1)}$, for $x, \alpha \in \mathbb{C}$, if $f(z) \in A(D)$, then we have on $D$ that

$$
W_{t}^{\alpha} f(z)=\mathbb{E} f\left(z e^{i B\left(\mathcal{E}_{\alpha}(t)\right)}\right)=\int_{\mathbb{R}} f\left(z e^{i u}\right) \ell_{\alpha}(u, t) d u=\sum_{k=0}^{\infty} a_{k} d_{k}(t) z^{k},
$$


where $d_{k}(t)=E_{\alpha}\left(-\frac{k^{2} t^{\alpha}}{2}\right), \alpha \in(0,1), \mathcal{E}_{\alpha}(t)$ is the inverse of the $\alpha$-stable subordinator and $\ell_{\alpha}$ is the probability density of the time-changed Brownian motion $\mathfrak{B}_{\alpha}(t):=B\left(\mathcal{E}_{\alpha}(t)\right)$.

Moreover, $W_{t}^{\alpha} f(z)$ is the unique solution $u(z, t)$ for the fractional Cauchy problem

$$
\begin{aligned}
& \frac{\partial^{\alpha} u}{\partial t^{\alpha}}(z, t)=\frac{1}{2} \frac{\partial^{2} u}{\partial \varphi^{2}}(z, t), \\
& (z, t) \in \mathbb{R}^{+} \times D, z=r e^{i \varphi}, r \in(0,1), \varphi \in[0,2 \pi) \\
& u(z, 0)=f(z), \quad z \in \bar{D}, f \in A(D) .
\end{aligned}
$$

Remark 2 Observing that, for $\alpha=1 / 2$ the following equality in distribution holds

$$
B\left(\mathcal{E}_{\alpha}(t)\right) \stackrel{d}{=} B_{1}\left(\left|B_{2}(t)\right|\right)
$$

where $B_{1}$ and $B_{2}$ are independent, we have that

$$
W_{t}^{1 / 2} f(z)=\mathbb{E} f\left(z e^{i B_{1}\left(\left|B_{2}(t)\right|\right)}\right)
$$

coincides with the solution to (4.16), for $\alpha=1 / 2$. Therefore, in this special case, we have an iterated Brownian motion on the circle.

Remark 3 In the stable case, i.e. for $g(\theta)=\theta^{\alpha}$, the inverse transform of the $r$-th moment given in (4.2) can be easily obtained and reads

$$
\mathbb{E}\left[\mathfrak{B}_{\alpha}(t)\right]^{r}=E_{\alpha}\left(-\frac{r}{2} t^{\alpha}\right), \quad r \in \mathbb{N} .
$$

Moreover, we can evaluate explicitly the mixed moment in (4.9), by recalling that $U_{g}(\tau)=\mathbb{E} \mathcal{E}_{\alpha}(\tau)=\tau^{\alpha} / \Gamma(\alpha+1)$ and that $\mathbb{E} \mathfrak{B}_{\alpha}(\tau)=E_{\alpha}\left(-\tau^{\alpha} / 2\right)$, so that, for $s<t$, we get

$$
\begin{aligned}
\mathbb{E}\left[\mathfrak{B}_{\alpha}(t) \overline{\mathfrak{B}_{\alpha}(s)}\right] & =E_{\alpha}\left(-\frac{t^{\alpha}}{2}\right)+\frac{\alpha}{2 \Gamma(\alpha+1)} \int_{0}^{s} E_{\alpha}\left(-\frac{(t-\tau)^{\alpha}}{2}\right) \tau^{\alpha-1} d \tau \\
& =E_{\alpha}\left(-\frac{t^{\alpha}}{2}\right)+\frac{t^{\alpha}}{2 \Gamma(\alpha)} \int_{0}^{s / t} E_{\alpha}\left(-\frac{t^{\alpha}(1-y)^{\alpha}}{2}\right) y^{\alpha-1} d y \\
& =E_{\alpha}\left(-\frac{t^{\alpha}}{2}\right)+\frac{t^{\alpha}}{2 \Gamma(\alpha)} \sum_{j=0}^{\infty} \frac{\left(-t^{\alpha} / 2\right)^{j}}{\Gamma(\alpha j+1)} B(\alpha j+1, \alpha ; s / t),
\end{aligned}
$$

where $B(a, b ; x):=\int_{0}^{x} z^{a-1}(1-z)^{b-1} d z$ is the incomplete beta function. Since, for $s \rightarrow t$, the previous expression reduces to one, we can write, for any $s, t \geq 0$,

$$
\mathbb{E}\left[\mathfrak{B}_{\alpha}(t) \overline{\mathfrak{B}_{\alpha}(s)}\right]=E_{\alpha}\left(-\frac{(t \vee s)^{\alpha}}{2}\right)
$$




$$
+\frac{(t \vee s)^{\alpha}}{2 \Gamma(\alpha)} \sum_{j=0}^{\infty} \frac{\left(-(t \vee s)^{\alpha} / 2\right)^{j}}{\Gamma(\alpha j+1)} B(\alpha j+1, \alpha ;(t \wedge s) /(t \vee s))
$$

Finally, it is easy to check that, for $\alpha=1,(4.8)$ reduces to $e^{-[t \vee s-t \wedge s] / 2}$ as it should be, since, in this case $\mathcal{E}_{\alpha}(t)=t$, a.s. for any $t \geq 0$.

\subsection{The tempered stable case}

For $g(\theta)=(\theta+\mu)^{\alpha}-\mu^{\alpha}$, for $\theta, \mu \geq 0, a=b=0$, and the process $\mathcal{E}_{g}(t)$ reduces to the inverse of the tempered stable subordinator (in the next $\mathcal{E}_{T}(t)$ ) and the operator $\mathfrak{D}_{t}^{g}$ coincides with the tempered derivative (see e.g. [2] and [3]) denoted by

$$
D_{t}^{\alpha, \mu} f(t):=e^{-\mu t} \frac{\partial^{\alpha}}{\partial t^{\alpha}}\left(e^{\mu t} f(t)\right)-\mu^{\alpha} f(t)=\left(\mu+\frac{d}{d t}\right)^{\alpha} f(t) .
$$

Since in this case the tail Lévy measure is given by

$$
w(s)=\frac{\alpha \mu^{\alpha} \Gamma(-\alpha, \mu s)}{\Gamma(1-\alpha)},
$$

where

$$
\Gamma(\rho, x)=\int_{x}^{+\infty} e^{-\omega} \omega^{\rho-1} d \omega,
$$

is the upper incomplete gamma function. The tempered derivative (4.19) can be also expressed in a convolution form, as follows

$$
D_{t}^{\alpha, \mu} f(t)=\frac{\alpha \mu^{\alpha}}{\Gamma(1-\alpha)} \int_{0}^{t} \frac{\partial}{\partial z} f(t-z) \Gamma(-\alpha, \mu z) d z .
$$

Corollary 2 Let $W_{t}^{g}$ be denoted as $W_{t}^{\alpha, \mu}$, for $g(\theta)=(\theta+\mu)^{\alpha}-\mu^{\alpha}$, then the complex integral

$$
W_{t}^{g} f(z)=\int_{\mathbb{R}} f\left(z e^{i u}\right) \ell_{\alpha, \mu}(u, t) d u=\sum_{k=0}^{\infty} a_{k} z^{k} d_{k}(t),
$$

where $\ell_{\alpha, \mu}$ is the probability density of the time-changed Brownian motion $B\left(\mathcal{E}_{T}(t)\right)$ and

$$
d_{k}(t)=\frac{1}{\Gamma(\alpha)} \int_{0}^{t} \Gamma(\alpha ; \mu(t-z)) \frac{e^{-\mu z}}{z} E_{\alpha, 0}\left(\left(\mu^{\alpha}-\frac{k^{2}}{2}\right) z^{\alpha}\right) d z
$$

is the unique solution of the fractional Cauchy problem

$$
\begin{aligned}
& D_{t}^{\alpha, \mu} u(z, t)=\frac{1}{2} \frac{\partial^{2} u}{\partial \varphi^{2}}(z, t), \\
& (z, t) \in D \times \mathbb{R}^{+}, \quad z=r e^{i \varphi}, r \in(0,1), \varphi \in[0,2 \pi)
\end{aligned}
$$




$$
u(z, 0)=f(z), \quad z \in \bar{D}, f \in A(D)
$$

Proof The result follows by using Theorem 1 and by inverting the Laplace transform

$$
\tilde{d}_{k}(\theta)=\frac{(\theta+\mu)^{\alpha}-\mu^{\alpha}}{\theta\left[(\theta+\mu)^{\alpha}-\mu^{\alpha}+\frac{k^{2}}{2}\right]} .
$$

This can be done by applying the well-known formula of the Laplace transform of the two-parameter Mittag-Leffler function (see e.g. [11]) and recalling that (see [4])

$$
\mathcal{L}\{\Gamma(\alpha ; \mu x) ; \theta\}=\frac{(\mu+\theta)^{\alpha}-\mu^{\alpha}}{\theta(\mu+\theta)^{\alpha}} .
$$

Remark 4 Note that, in the special case $\mu=0$, the expression of $d_{k}(t)$ reduces, for any $k \in \mathbb{N}$ and $t \geq 0$ to

$$
d_{k}(t)=\int_{0}^{t} \frac{1}{z} E_{\alpha, 0}\left(-\frac{k^{2}}{2} z^{\alpha}\right) d z=E_{\alpha, 1}\left(-\frac{k^{2}}{2} t^{\alpha}\right),
$$

which coincides with the stable case.

\section{Higher dimensional extensions}

Following [8,9], we can also consider the $n$-dimensional fractional Cauchy problem extending (3.12)-(3.13). In this case, the probabilistic interpretation of the solution for the complexified multidimensional fractional heat equation can be obtain by means of $n$-dimensional Brownian motions time changed with the inverse of the subordinator $\left\{\mathcal{E}_{g}(t), t \geq 0\right\}$.

Let $z_{1}, z_{2}, \ldots, z_{n} \in \bar{D}$ and $f\left(z_{1}, z_{2}, \ldots, z_{n}\right) \in A\left(D^{n}\right)$, which means that $f\left(z_{1}, z_{2}, \ldots, z_{n}\right)$ belongs to $A(D)$ for each complex variable $z_{1}, z_{2}, \ldots, z_{n}$. Let us deal with the arguments developed in Section 3 and extend them to the multidimensional case. Let us introduce the following complex integral

$$
\begin{array}{rl}
\bar{W}_{t}^{g} & f\left(z_{1}, z_{2}, \ldots, z_{n}\right) \\
& =\frac{1}{(2 \pi)^{n / 2}} \int_{-\infty}^{+\infty} \cdots \int_{-\infty}^{+\infty} f\left(z_{1} e^{-i u_{1}}, \ldots z_{n} e^{-i u_{n}}\right) d u_{1} \cdots d u_{n} \\
& \times \int_{0}^{+\infty} \frac{e^{-\frac{u_{1}^{2}+u_{2}^{2} \cdots+u_{n}^{2}}{2 y}}}{y^{n / 2}} m_{g}(y, t) d y \\
& =\mathbb{E} f\left(z_{1} e^{-i B_{1}\left(\mathcal{E}_{g}(t)\right)}, z_{2} e^{-i B_{2}\left(\mathcal{E}_{g}(t)\right)}, \ldots, z_{n} e^{-i B_{n}\left(\mathcal{E}_{g}(t)\right)}\right) \\
& =\mathbb{E} f\left(\mathfrak{B}_{1, g}^{z_{1}}(t), \mathfrak{B}_{2, g}^{z_{2}}(t), \ldots, \mathfrak{B}_{n, g}^{z_{n}}(t)\right),
\end{array}
$$


for $z_{1}, z_{2}, \cdots, z_{n} \in \bar{D}$. Furthermore, $\mathfrak{B}_{k, g}^{z_{k}}(t):=z_{k} e^{-i B_{k}\left(\mathcal{E}_{g}(t)\right)}$ with $z_{k}=r_{k} e^{i \varphi_{k}}, r_{k} \in$ $(0,1], \varphi_{k} \in[0,2 \pi)$, and $\left\{B_{k}(t), t \geq 0\right\}$, are independent standard Brownian motion for $k=1,2, \ldots, n$. Therefore, the time-changed process

$$
\mathfrak{B}_{g}^{z_{1}, \ldots, z_{n}}:=\left\{\left(\mathfrak{B}_{1, g}^{z_{1}}(t), \mathfrak{B}_{2, g}^{z_{2}}(t), \ldots, \mathfrak{B}_{n, g}^{z_{n}}(t)\right), t \geq 0\right\}
$$

represents a $n$-dimensional wrapped Brownian motion on the $n$-dimensional circle $\mathbb{S}^{n}:=\mathbb{S}_{r_{1}} \times \cdots \times \mathbb{S}_{r_{n}}$, where the coordinate processes are the circular Brownian motions (3.9) with random time $\mathcal{E}_{g}(t)$ (and then they are not independent). Therefore, simple calculations show that $\mathfrak{B}_{g}^{z_{1}, \ldots, z_{n}}$ has covariance matrix with entries

$$
\begin{aligned}
q_{i, j} & =\mathbb{E}\left[\mathfrak{B}_{i, g}^{z_{i}}(t) \overline{\mathfrak{B}_{j, g}^{z_{j}}(t)}\right]-\mathbb{E}\left[\mathfrak{B}_{i, g}^{\left.z_{i}(t)\right]} \overline{\mathbb{E}\left[\overline{\mathfrak{B}_{j, g}^{z_{j}}(t)}\right]}\right. \\
& = \begin{cases}z_{i} \bar{z}_{j}\left\{\mathbb{E}\left[e^{-2 \mathcal{E}_{g}(t)}\right]-\left(\mathbb{E}\left[e^{-\frac{\mathcal{E}_{g}(t)}{2}}\right]\right)^{2}\right\}, & i \neq j, \\
z_{i} \bar{z}_{i}\left\{1-\left(\mathbb{E}\left[e^{-\frac{\mathcal{E}_{g}(t)}{2}}\right]\right)^{2}\right\}, & i=j .\end{cases}
\end{aligned}
$$

Now, we recall that, by the multivariate Taylor's expansion, we can write

$$
f\left(z_{1}, \ldots, z_{n}\right)=\sum_{k_{1}, \ldots, k_{n}=0}^{\infty} a_{k_{1}, \ldots, k_{n}} z_{1}^{k_{1}} \cdots z_{n}^{k_{n}}
$$

Then, we have the following result representing the multidimensional version of Theorem 1.

Theorem 5 (i) If $f \in A\left(D^{n}\right)$, then we have that the complex integral (5.1) can be written as

$$
\bar{W}_{t}^{g} f\left(z_{1}, z_{2}, \ldots, z_{n}\right)=\sum_{k_{1}, \ldots, k_{n}=0}^{\infty} a_{k_{1}, \ldots, k_{n}} z_{1}^{k_{1}} \cdots z_{n}^{k_{n}} d_{k_{1}, \ldots, k_{n}}(t)
$$

where $d_{k_{1}, \ldots, k_{n}}(t)=\mathbb{E}\left[e^{-\frac{\mathcal{E}_{g}(t)}{2} \sum_{j=1}^{n} k_{j}^{2}}\right]$. Furthermore

$$
\begin{aligned}
R_{\theta} \bar{W}_{t}^{g} f\left(z_{1}, z_{2}, \ldots, z_{n}\right) & :=\int_{0}^{\infty} e^{-\theta t} \bar{W}_{t}^{g} f\left(z_{1}, z_{2}, \ldots, z_{n}\right) d t \\
& =\sum_{k_{1}, \ldots, k_{n}=0}^{\infty} a_{k_{1}, \ldots, k_{n}} z_{1}^{k_{1}} \cdots z_{n}^{k_{n}} \tilde{d}_{k_{1}, \ldots, k_{n}}(t),
\end{aligned}
$$

where

$$
\tilde{d}_{k_{1}, \ldots, k_{n}}(\theta)=\frac{g(\theta) / \theta}{g(\theta)+\left(k_{1}^{2}+\ldots+k_{n}^{2}\right) / 2}
$$


(ii) Let $t>0, f \in A\left(D^{n}\right)$ and $z_{1}, \ldots, z_{n} \in \bar{D}$. The integral operator (5.1) is the unique solution

$$
u\left(z_{1}, z_{2}, \ldots, z_{n}, t\right)=\bar{W}_{t}^{g} f\left(z_{1}, z_{2}, \ldots, z_{n}\right)
$$

(that belongs to $A(D)$ for each complex variable $z_{k}$ ) of the fractional Cauchy problem

$$
\begin{aligned}
& \mathfrak{D}_{t}^{g} u\left(z_{1}, z_{2}, \ldots, z_{n}, t\right)=\frac{1}{2}\left[\frac{\partial^{2}}{\partial \varphi_{1}^{2}}+\ldots+\frac{\partial^{2}}{\partial \varphi_{n}^{2}}\right] u\left(z_{1}, z_{2}, \ldots, z_{n}, t\right), \\
& z_{1}=r_{1} e^{i \varphi_{1}}, \ldots, z_{n}=r_{n} e^{i \varphi_{n}} \in D \backslash\{0\} \\
& u\left(z_{1}, z_{2}, \ldots, z_{n}, 0\right)=f\left(z_{1}, z_{2}, \ldots, z_{n}\right),
\end{aligned}
$$

where $\varphi_{k}$ is the principal value of $z_{k}$.

Proof (i) The representation (5.2) follows by writing (5.1) as follows

$$
\begin{aligned}
\bar{W}_{t}^{g} f\left(z_{1}, z_{2}, \cdots, z_{n}\right) \\
=\sum_{k_{1}, \ldots, k_{n}=0}^{\infty} a_{k_{1}, \ldots, k_{n}} z_{1}^{k_{1}} \cdots z_{n}^{k_{n}} \int_{-\infty}^{+\infty} d u_{n} \ldots \int_{-\infty}^{+\infty} d u_{1} e^{-i \sum_{j=1}^{n} u_{j} k_{j}} \\
\quad \times \int_{0}^{+\infty} \frac{e^{-\frac{u_{1}^{2}+u_{2}^{2} \ldots+u_{n}^{2}}{2 y}}}{(2 \pi y)^{n / 2}} m_{g}(y, t) d y \\
=\sum_{k_{1}, \ldots, k_{n}=0}^{\infty} a_{k_{1}, \ldots, k_{n}} z_{1}^{k_{1}} \cdots z_{n}^{k_{n}} \mathbb{E}\left[e^{-i \sum_{j=1}^{n} k_{j} B_{j}\left(\mathcal{E}_{g}(t)\right)}\right] \\
=\sum_{k_{1}, \ldots, k_{n}=0}^{\infty} a_{k_{1}, \ldots, k_{n}} z_{1}^{k_{1}} \cdots z_{n}^{k_{n}} \mathbb{E}\left[e^{-\frac{\mathcal{E}_{g}(t)}{2} \sum_{j=1}^{n} k_{j}^{2}}\right] .
\end{aligned}
$$

Since the time-Laplace transform of $\mathbb{E} e^{-i \sum_{j=1}^{n} k_{j} B_{j}\left(\mathcal{E}_{g}(t)\right)}$ coincides with (5.4) (see, for example, [5]), the result (5.3) holds true.

(ii) Analogously to the one-dimensional case, we take the time-Laplace transform of both sides of (5.5), as follows

$$
\begin{aligned}
\mathcal{L} & \left\{D_{t}^{g} \bar{W}_{t}^{g}(f)\left(z_{1}, \ldots, z_{n}\right) ; \theta\right\} \\
& =\frac{g(\theta)}{\theta}\left[\sum_{k_{1}=0, \ldots, k_{n}=0}^{\infty} \frac{a_{k_{1}, \ldots k_{n}} z_{1}^{k_{1}} \ldots z_{n}^{k_{n}} g(\theta)}{g(\theta)+\left(k_{1}^{2}+\ldots+k_{n}^{2}\right) / 2}-f\left(z_{1}+\ldots+z_{n}\right)\right]
\end{aligned}
$$

and

$$
\frac{1}{2}\left[\frac{\partial^{2}}{\partial \varphi_{1}^{2}}+\ldots+\frac{\partial^{2}}{\partial \varphi_{n}^{2}}\right] \mathcal{L}\left\{\bar{W}_{t}^{g}(f)\left(z_{1}, \ldots, z_{n}\right) ; \theta\right\}
$$




$$
=-\frac{1}{2} \sum_{k_{1}=0, \ldots, k_{n}=0}^{\infty} \frac{g(\theta) / \theta}{\frac{\left(k_{1}^{2}+\ldots+k_{n}^{2}\right)}{2}+g(\theta)} a_{k_{1}, \ldots, k_{n}}\left(k_{1}^{2}+\ldots+k_{n}^{2}\right) z_{1}^{k_{1}} \cdots z_{n}^{k_{n}} .
$$

Therefore (5.7), (5.8) and (5.2) allow to conclude the proof.

Open Access This article is licensed under a Creative Commons Attribution 4.0 International License, which permits use, sharing, adaptation, distribution and reproduction in any medium or format, as long as you give appropriate credit to the original author(s) and the source, provide a link to the Creative Commons licence, and indicate if changes were made. The images or other third party material in this article are included in the article's Creative Commons licence, unless indicated otherwise in a credit line to the material. If material is not included in the article's Creative Commons licence and your intended use is not permitted by statutory regulation or exceeds the permitted use, you will need to obtain permission directly from the copyright holder. To view a copy of this licence, visit http://creativecommons.org/licenses/by/4.0/.

\section{References}

1. Applebaum, D.: Lévy Processes and Stochastic Calculus. Cambridge University Press, Cambridge (2009)

2. Baeumer, B., Meerschaert, M.M.: Tempered stable Lévy motion and transient super-diffusion. J. Comput. Appl. Math. 233(10), 2438-2448 (2010)

3. Beghin, L.: On fractional tempered stable processes and their governing differential equations. J. Comput. Phys. 293, 29-39 (2015)

4. Beghin, L., Gajda, J.: Tempered relaxation equation and related generalized stable processes. Fract. Calc. Appl. Anal. 23(5), 1248-1273 (2020). https://doi.org/10.1515/fca-2020-0063

5. Beghin, L., Macci, C., Ricciuti, C.: Random time-change with inverses of multivariate subordinators: governing equations and fractional dynamics. Stoch. Process. Appl. 130, 6364-6387 (2020)

6. Chen, Z.Q.: Time fractional equations and probabilistic representation. Chaos Solit. Fract. 102, 168174 (2017)

7. Feller, W.: An Introduction to Probability Theory and Its Applications, vol. II. Wiley, New YorkLondon-Sydney (1966)

8. Gal, C.G., Gal, S.G., Goldstein, J.A.: Evolution equations with real time variable and complex spatial variables. Compl. Variabl. Ellipt. Equ. 53(8), 753-74 (2008)

9. Gal, C.G., Gal, S.G., Goldstein, J.A.: Evolution Equations with a Complex Spatial Variable, vol. 14. World Scientific, Singapore (2014)

10. Giusti, A.: General fractional calculus and Prabhakar's theory. Commun. Nonlin. Sci. Numer. Simul. 83, 105114 (2020)

11. Gorenflo, R., Kilbas, A.A., Mainardi, F., Rogosin, S.V.: Mittag-Leffler Functions, Related Topics and Applications, 2nd edn. Springer, Berlin (2014)

12. Kilbas, A.A., Srivastava, H.M., Trujillo, J.J.: Theory and Applications of Fractional Differential Equations, vol. 204. Elsevier Science Ltd, Amsterdam (2006)

13. Kochubei, A.N.: General fractional calculus, evolution equations, and renewal processes. Integr. Equ. Oper. Theory 71, 583-600 (2011)

14. Kolokoltsov, V.: The probabilistic point of view on the generalized fractional partial differential equations. Fract. Calc. Appl. Anal. 22(3), 543-600 (2019). https://doi.org/10.1515/fca-2019-0033

15. Mainardi, F., Mura, A., Pagnini, G.: The $M$-Wright function in time-fractional diffusion processes: a tutorial survey. J. Differ. Equ. 2010, 104505 (2010)

16. Mardia, K.V., Jupp, P.E.: Directional Statistics. Wiley Ser. in Probability and Statistics, Wiley, Chichester (2000)

17. Meerschaert, M.M., Scheffler, H.P.: Limit theorems for continuous time random walks with infinite mean waiting times. J. Appl. Probab. 41, 623-638 (2004)

18. Meerschaert, M.M., Scheffler, H.P.: Triangular array limits for continuous time random walks. Stoch. Proc. Applic. 118, 1606-1633 (2008) 
19. Meerschaert, M.M., Sikorskii, A.: Stochastic Models for Fractional Calculus. De Gruyter, BerlinBoston (2012)

20. Meerschaert, M.M., Straka, P.: Inverse stable subordinators. Math. Model. Nat. Phenom. 8(2), 1-16 (2013)

21. Meerschaert, M.M., Toaldo, B.: Relaxation patterns and semi-Markov dynamics. Stoch. Proc. Applic. 129(8), 2850-2879 (2019)

22. Schilling, R.L., Song, R., Vondracek, Z.: Bernstein Functions. Walter De Gruyter, Berlin (2012)

23. Toaldo, B.: Convolution-type derivatives, hitting-times of subordinators and time-changed $C_{0^{-}}$ semigroups. Potent. Anal. 42(1), 115-140 (2015)

24. Veillette, M., Taqqu, M.S.: Using differential equations to obtain joint moments of first-passage times of increasing Lévy processes. Stat. Probab. Lett. 80(7-8), 697-705 (2010)

25. Whitt, W.: Stochastic-Process Limits. Springer, New York (2002)

Publisher's Note Springer Nature remains neutral with regard to jurisdictional claims in published maps and institutional affiliations. 